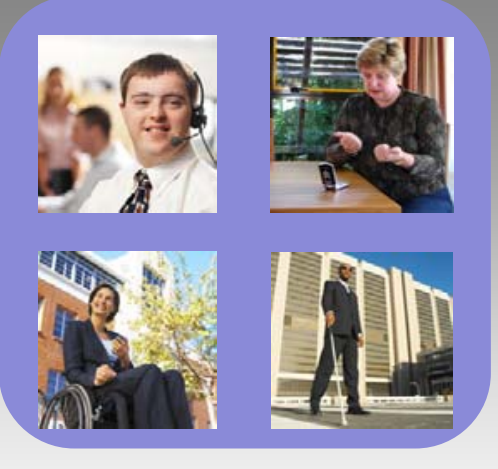

\title{
Risk Factors Associated with the Professional Performance of Civil Servants with Impairment
}

\section{Belén Rando \& Cláudia Anjos}

National Institute of Administration (Portugal) 


\section{POLICY OF HEALTH AND SAFETY AT WORK}

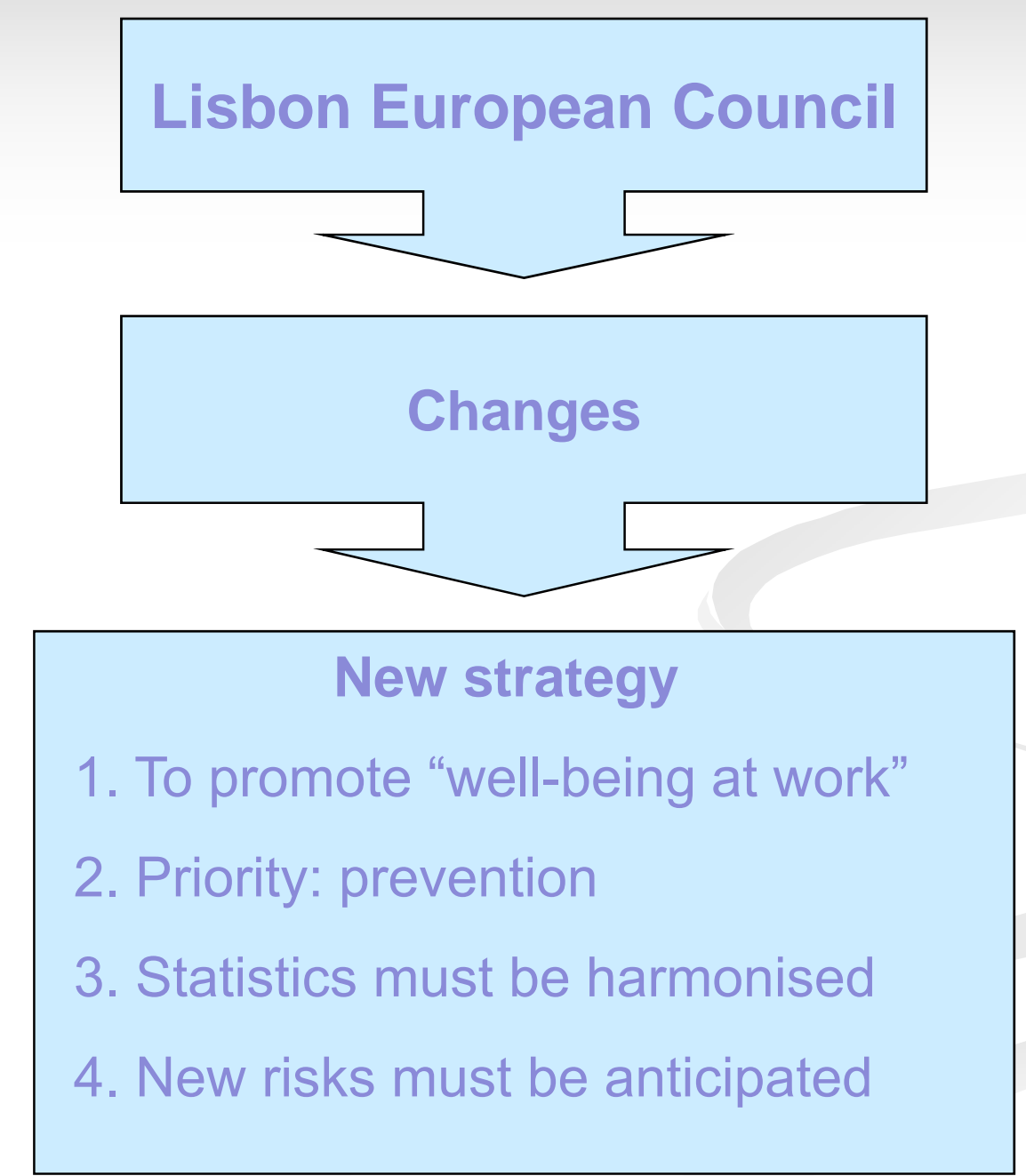




\section{WHAT IS AN IMPAIRMENT?}

ICF (WHO, 2001):

IMPAIRMENTS ARE PROBLEMS IN BODY FUNCTION (INCLUDING PSYCHOLOGICAL FUNCTIONS) OR BODY STRUCTURE SUCH AS A SIGNIFICANT DEVIATION OR LOSS. 


\section{POLICY OF PEOPLE WITH IMPAIRMENT}

\section{UNO}

- R99 - vocational rehabilitation of the disabled, Recommendation (1955)

- International Year of Disabled Persons (1981)

- R168 - Vocational Rehabilitation and Employment, Recommendation (1983)

- C159 Vocational Rehabilitation and Employment, Convention (1983)

- Resolution 45/91 - "Society for All" (1990)

- Resolution 48/96 - Standard Rules on the Equalization of Opportunities for Persons with Disabilities (1993)

- World Summit on the Information Society (2002) 


\section{POLICY OF PEOPLE WITH IMPAIRMENT}

\section{EUROPE}

- Turim Treaty or European Social Charter (1961)

- Recommendation R(92)6 - Coherent Policy for People with Disabilitites

- Communication COM(96)406 final - Equality of Opportunity for People with Disabilities

- Directive 2000/78/EEC - Equality in employment and profissional activity

- ResAP (2001)3 - Full Citizenship of People with Disabilities Through Inclusive Technologies

- Resolution 2003/C 39/03 - improving eAccessibility for people with disabilities

eEurope 2002 e eEurope 2005

- European Year of People with Disabilities (2003)

- Equal opportunities for people with disabilities: an European action plan (2004-2010)

European Year of Equal Opportunities for All (2007) 


\section{POLICY OF PEOPLE WITH IMPAIRMENT}

\section{POR'TUGAL}

Constitution of the Portuguese Republic (1976)

Act 9/89 - Law on Prevention, Rehabilitation and Integration of People with Disabilities

Resolution 6/98 - Green Book of the Information Society

- Resolution 96/99 - National Initiative for Citizens with Special Needs in the IS

Law 99/2003 - Labour Code

Resolution 109/2003 - National Initiative for the Broadband

Decree Law 163/2006 - accessibility to buildings and public via

Resolution 120/2006 - Action Plan for Integration of People with Impairment or Disability

Law 46/2006 - prohibition and punishment of discrimination

Resolution 9/2007 - National Plan for accessibility 


\section{THEORETICAL MODEL}

BIOPSYCHOSOCIAL MODEL ICF (WHO, 2001); SOCIAL INCLUSION MODEL (WERNECK, 2005)

\section{HEALTH CONDITION}

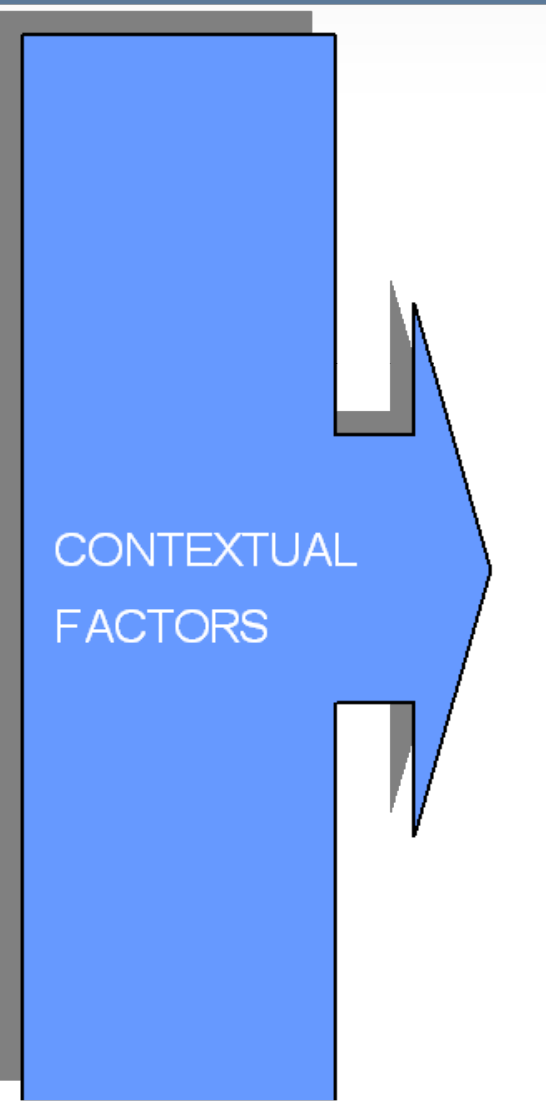

\section{PERSONAL FACTORS}

Gender, age, coping styles, etc.

ENVIRONMENTAL FACTORS

ACESSIBILITY:

\section{Architectural}

Communicational

Methodological

Instrumental (tools)

Policy planning

Attitudinal

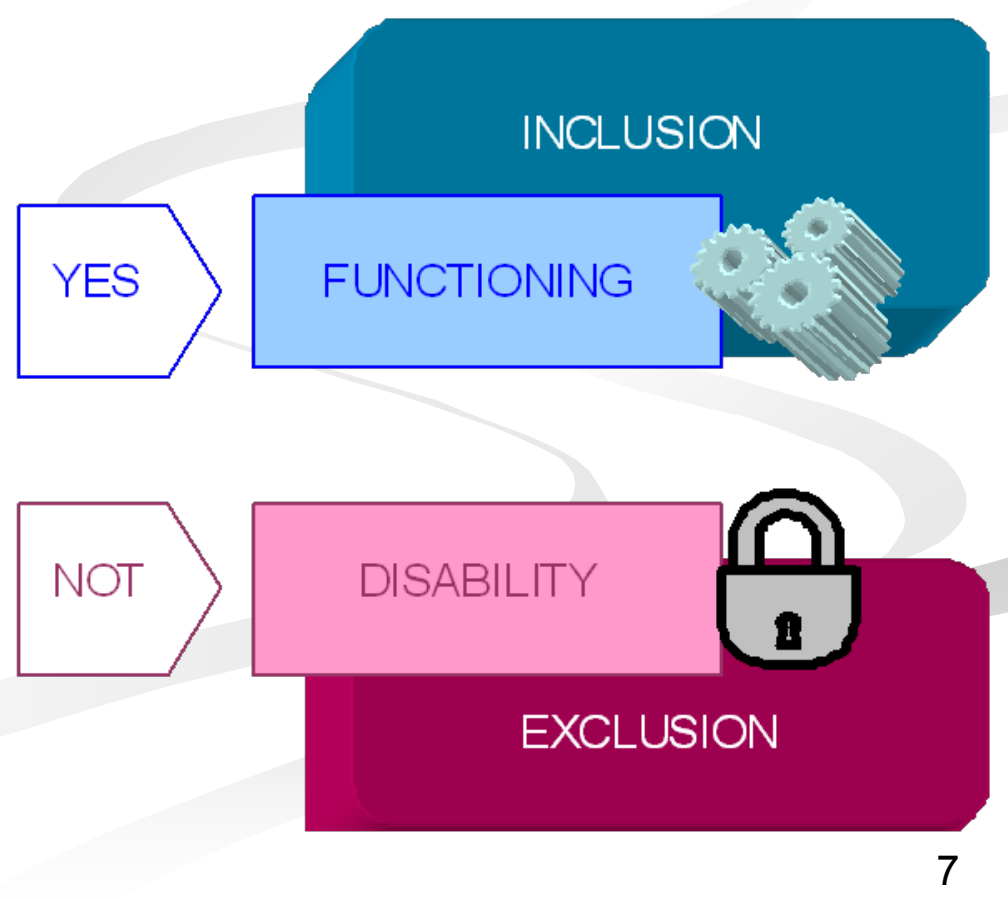




\section{OBJECTIVE}

\section{GENERAL}

To know labour conditions of civil servants with impairment who work in the Portuguese Central Public Administration

\section{SPECIFIC}

To know risk factors associated with their professional performance 


\section{METHOD}

\section{PARTICIPANTS ( $n=980$ civil servants)}

- $\quad 94.6 \%>6$ years in Public Administration

- $\quad 74.5 \%$ with impairment after joining to Public Administration

- More frequent: Musculoskeletal impairment, cancer \& visual impairment

- $33.4 \%$ two or more impairments

- $\quad 7.9 \%$ work accident 


\section{METHOD}

\section{PARTICIPANTS ( $n=980$ civil servants)}

- $\quad 54 \%$ women; $46 \%$ men

- $41.6 \%$ aged 35 to $50 ; 48.5 \%$ aged 51 to $64(M=50,77 ; S D=8,57)$

- $\quad 30.5 \%$ Univ. degree; $21.9 \%$ Primary Educ.; 18.4\% Secondary Educ.

- $\quad 23.1 \%$ Administrative Career; $17.6 \%$ Security Forces; $16.7 \%$ Auxiliary Career

- $\quad 39 \%$ Ministry of Health; $18.1 \%$ Ministry of the Interior 


\section{METHOD}

\section{INSTRUMENT}

Questionnaire (38 questions; mainly multiple-choice)

- Impairment Profile

- Occupational Context

Socio-professional factors

Environmental factors 


\section{METHOD}

\section{PROCEDURE}

Delivered to 204 ministerial organizations (5th June-31st August)
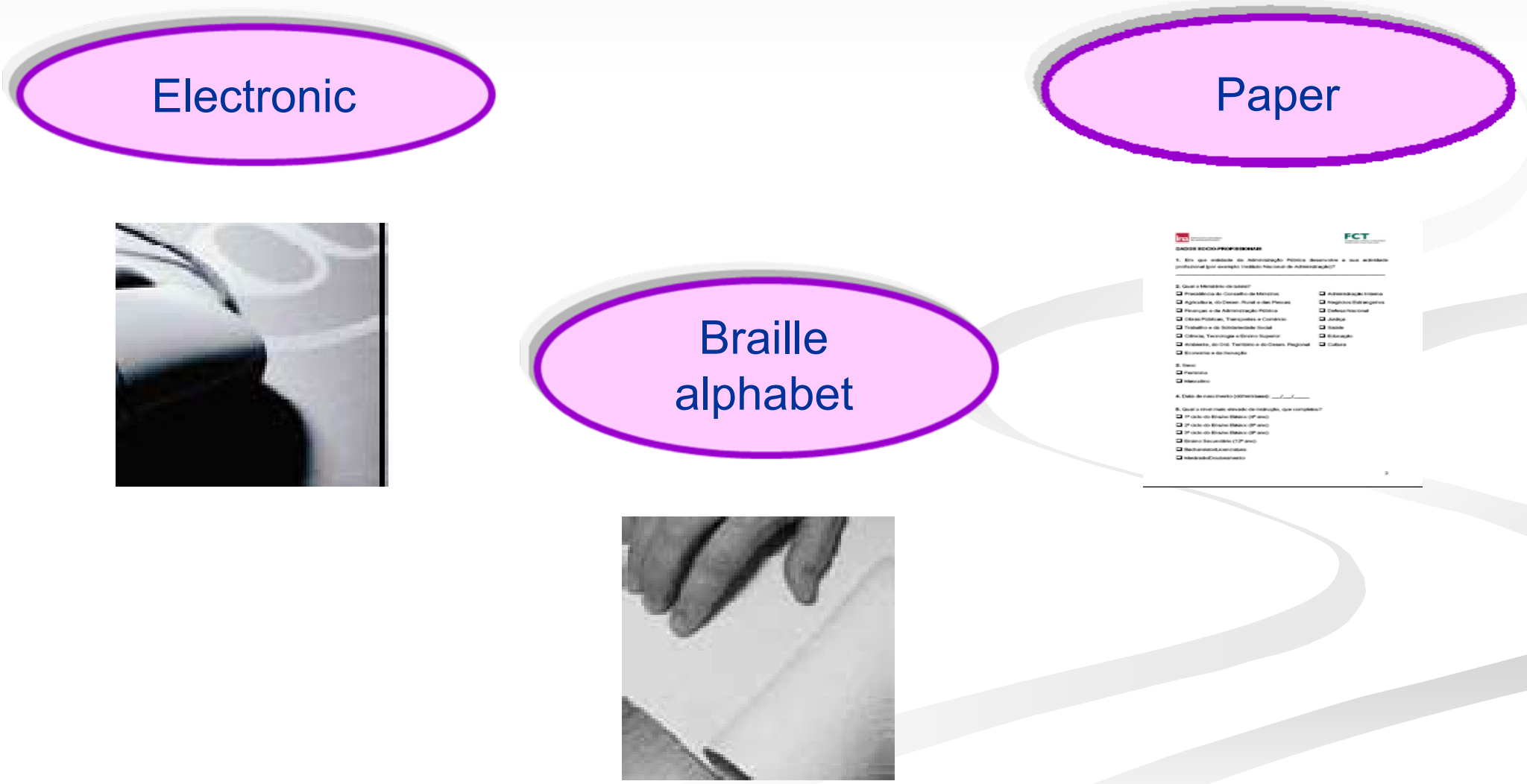


\section{RESULTS}

\section{WORKPLACE ACCESSIBILITY: ENTRANCE \& SPACES}

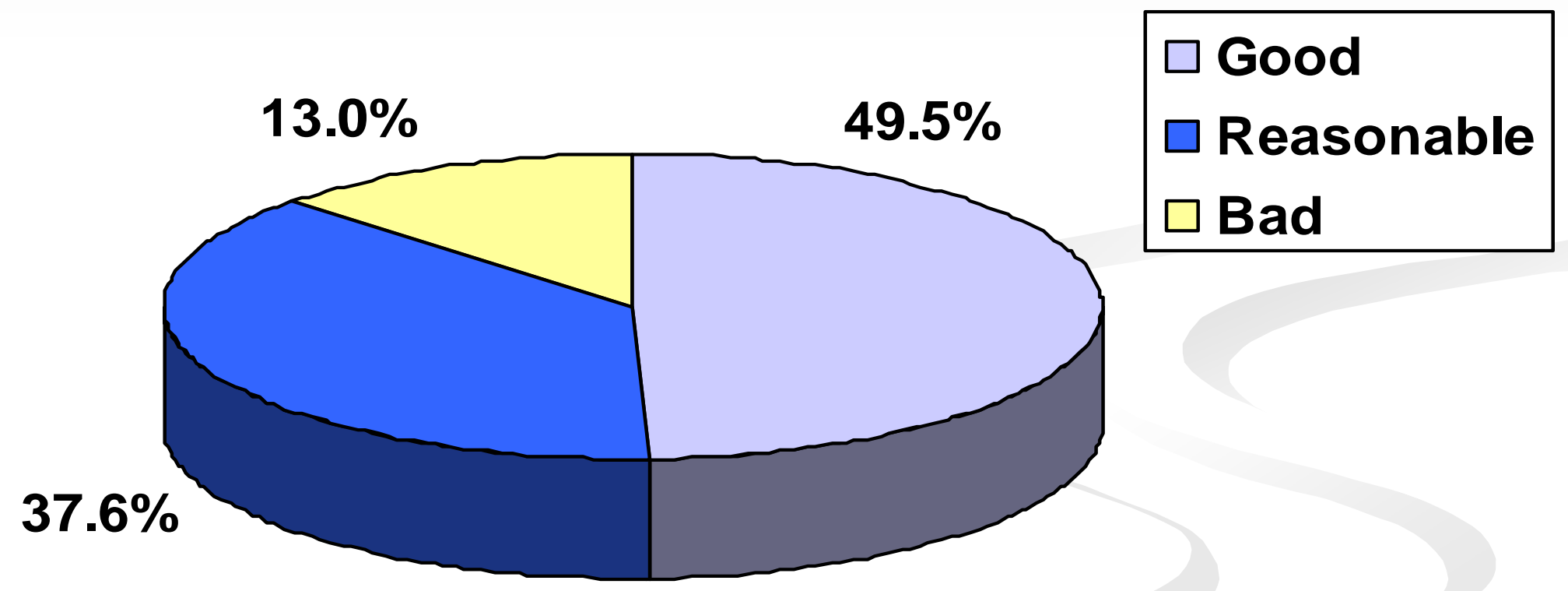




\section{RESULTS}

\section{WORKPLACE ACCESSIBILITY: OWN SPACE}

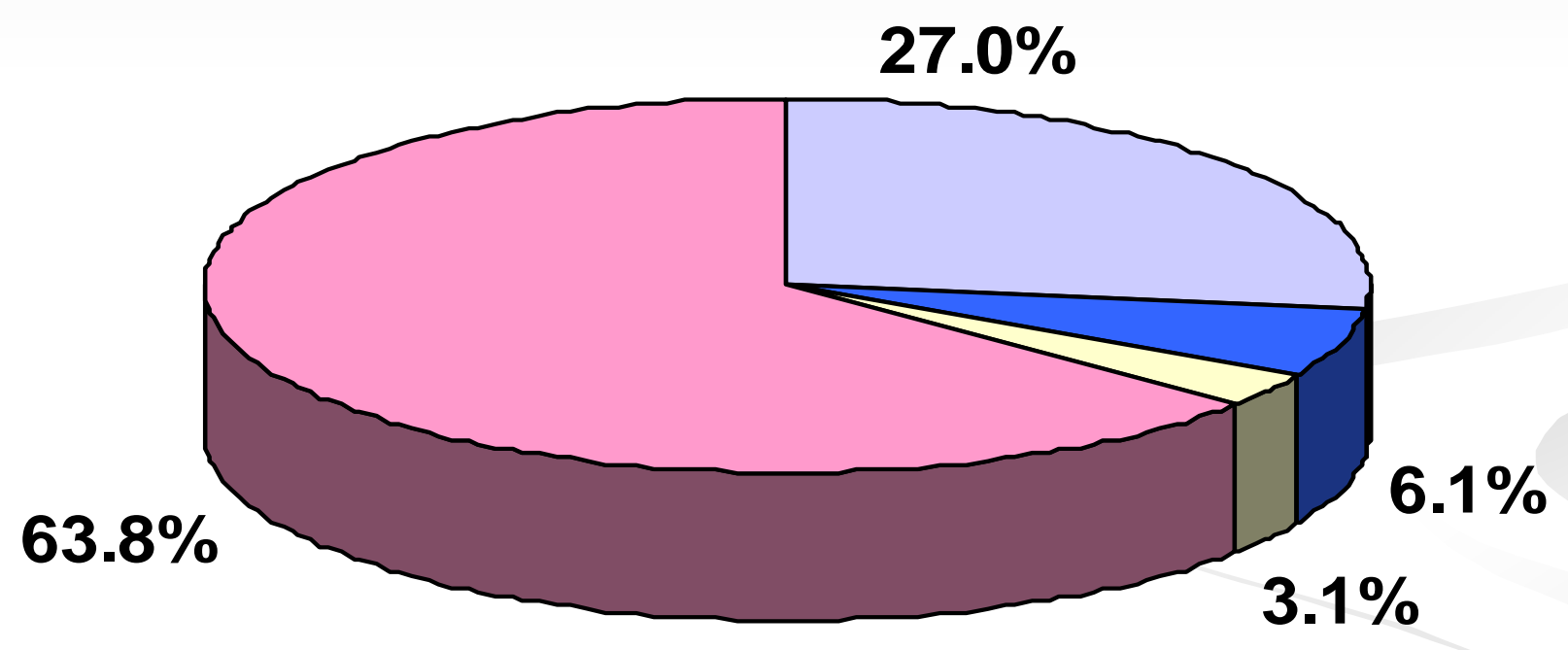

$\square$ Done and adequate

$\square$ Done and inadequate

$\square$ Being done $\square$ Necessary to be done 


\section{RESULTS}

\section{COMPUTER}

\section{$20 \%$ haven't a computer}

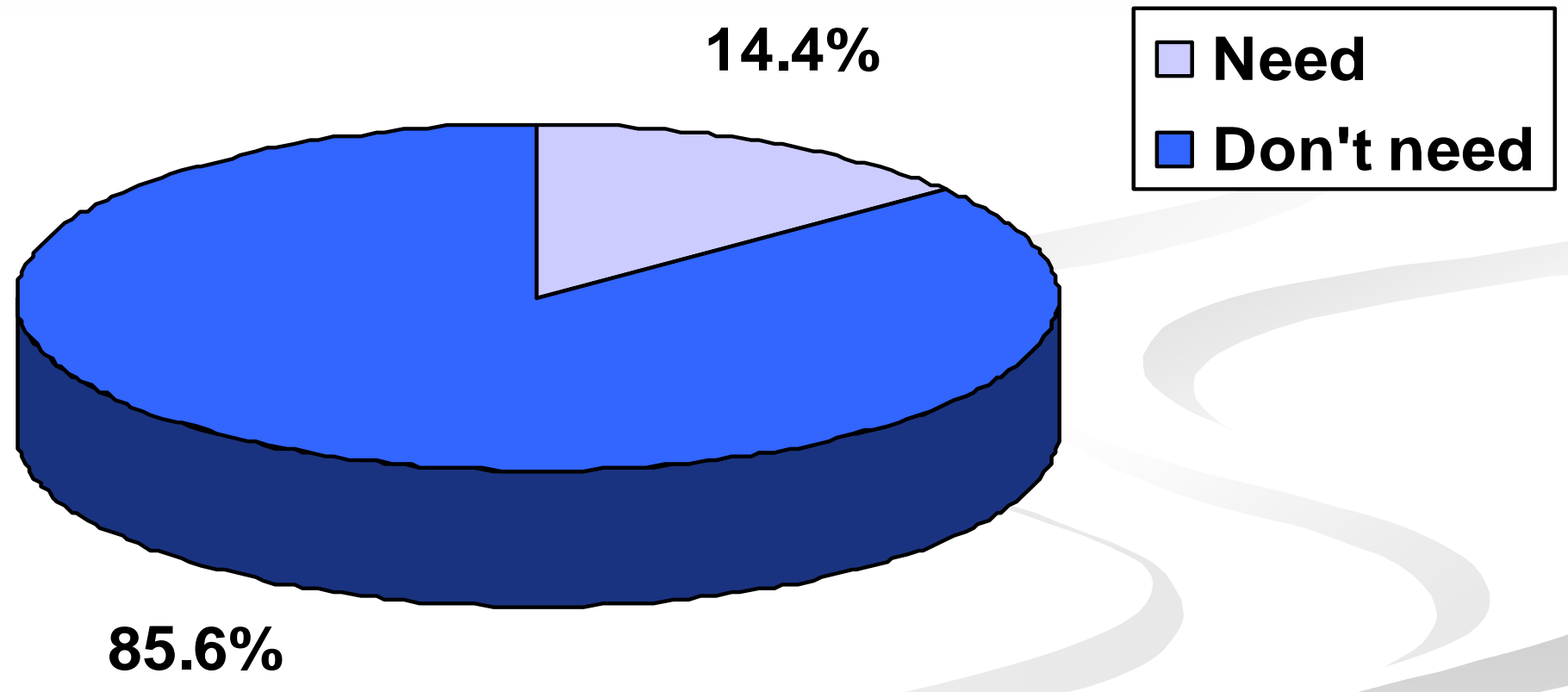




\section{RESULTS}

\section{INTERNET ACCESS}

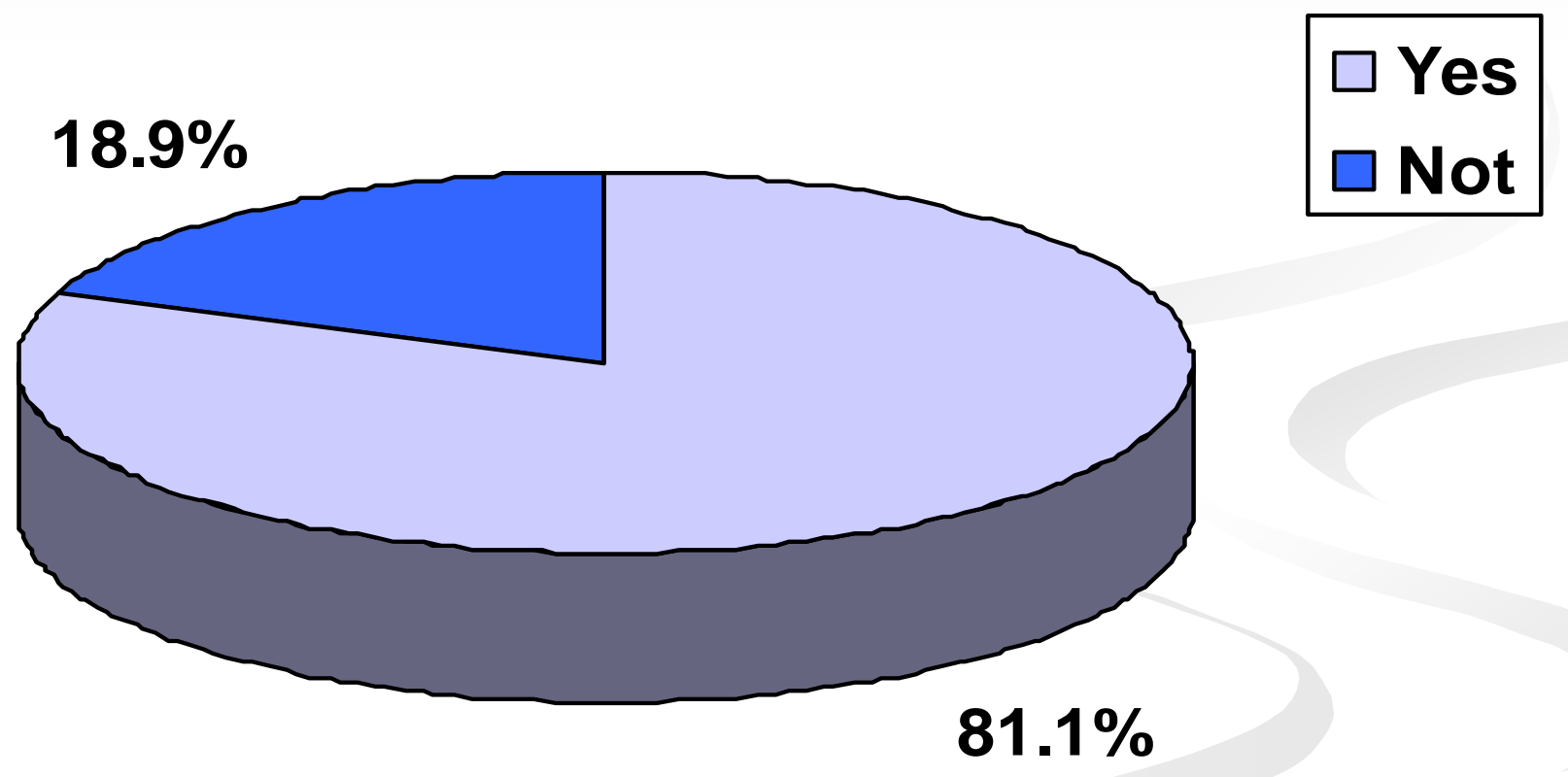




\section{RESULTS}

\section{SOFTWARE ACCESSIBILITY}

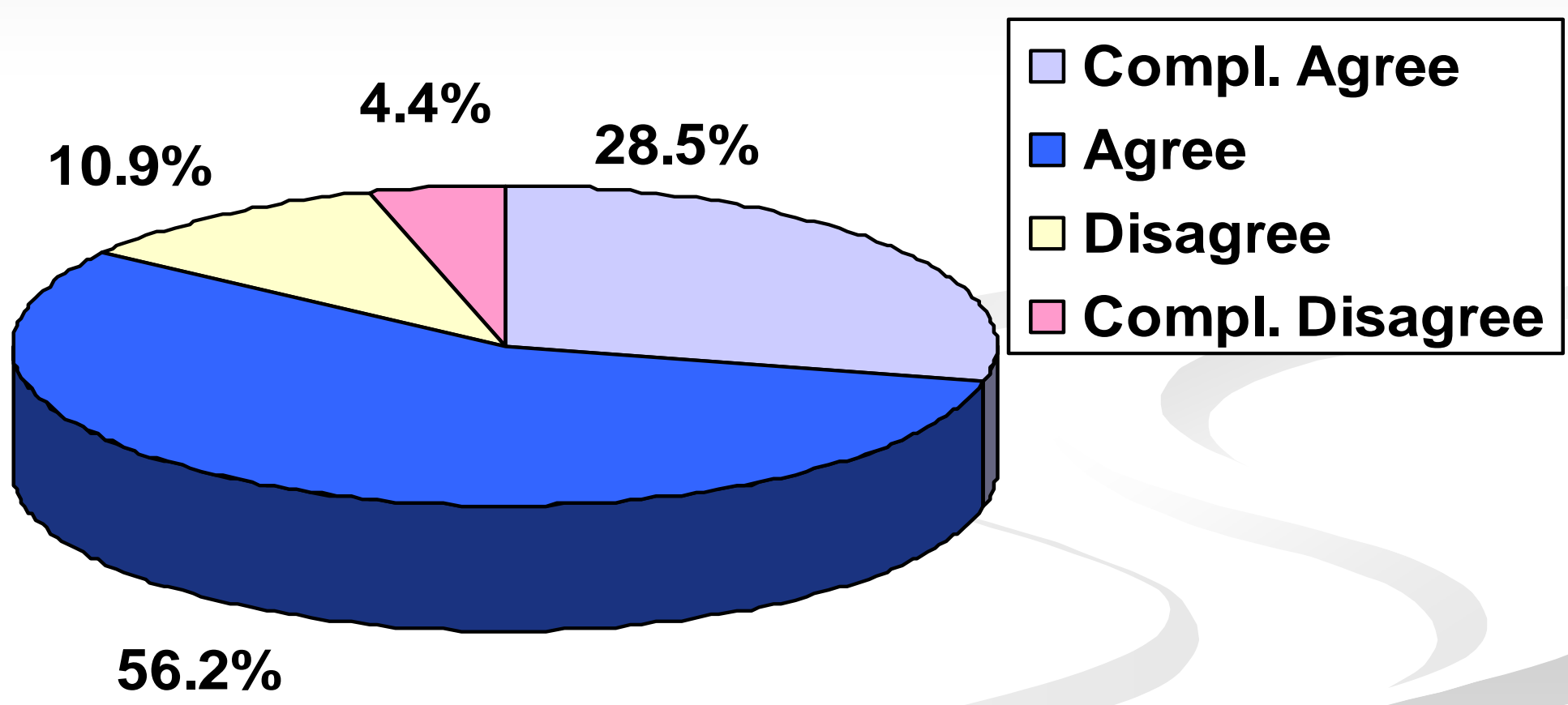




\section{RESULTS}

\section{ASSISTIVE TECHNOLOGIES}

\begin{tabular}{|l|c|c|}
\hline It's necessary & Don't Have & Career \\
\hline Notepad $(n=11)$ & $64.0 \%$ & Auxiliary \\
\hline Braille Line $(n=18)$ & $39.0 \%$ & Auxiliary \\
\hline MG/MG TV $(n=17)$ & $71.0 \%$ & Executive Officer \\
\hline Speech Synthesizer $(n=31)$ & $45.0 \%$ & Auxiliary \\
\hline Large Monitor $(n=22)$ & $73.0 \%$ & Administrative \\
\hline Enlargement Software $(n=19)$ & $79.0 \%$ & Administrative \\
\hline
\end{tabular}




\section{RESULTS}

\section{PERCEPTIONS ABOUT COWORKERS AND MANAGER}

\begin{tabular}{|l|c|l|c|}
\hline COWORKERS & Valid \% & MANAGER & Valid \% \\
\hline Valued & 42.7 & Valued & 43.4 \\
\hline $\begin{array}{l}\text { Neither valued, } \\
\text { nor devalued }\end{array}$ & 42.9 & $\begin{array}{l}\text { Neither valued, } \\
\text { nor devalued }\end{array}$ & 43.1 \\
\hline Devalued & 4.0 & Devalued & 3.4 \\
\hline Don't know & 10.4 & Don't know & 10.1 \\
\hline Total valid & 100.0 & Total valid & 100.0 \\
\hline
\end{tabular}




\section{RESULTS}

\section{PERCEPTIONS ABOUT COWORKERS AND MANAGER: AWARENESS}

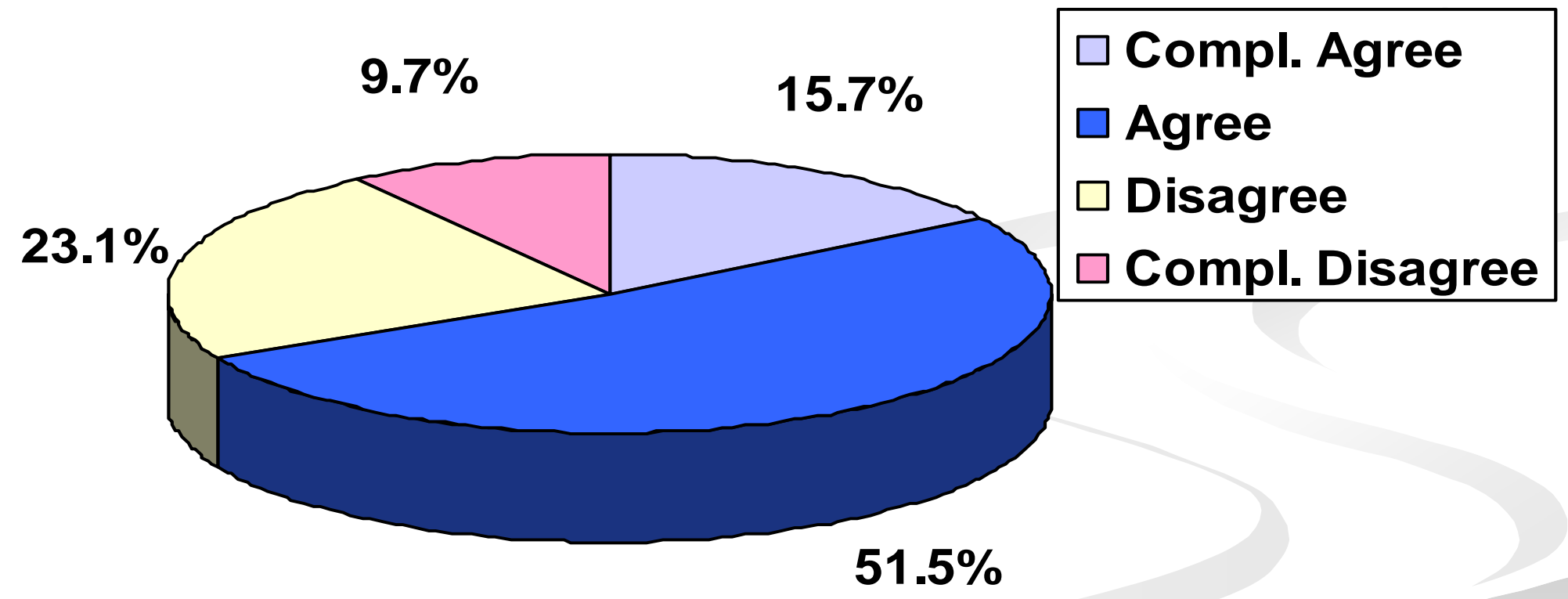




\section{DISCUSSION}

1. Inadequate working conditions

2. Insufficient awareness

3. Direct and indirect discrimination

4. Assessment by objectives?

5. Restructuring Programme of Central Administration (PRACE)

6. Minimum salary $=410 € ;$ only Executive Officer $\geq 1200 €$ 


\section{DISCUSSION}

DIMENSIONS OF PRECARIOUS WORK (Rodgers \& Rodgers,1989):

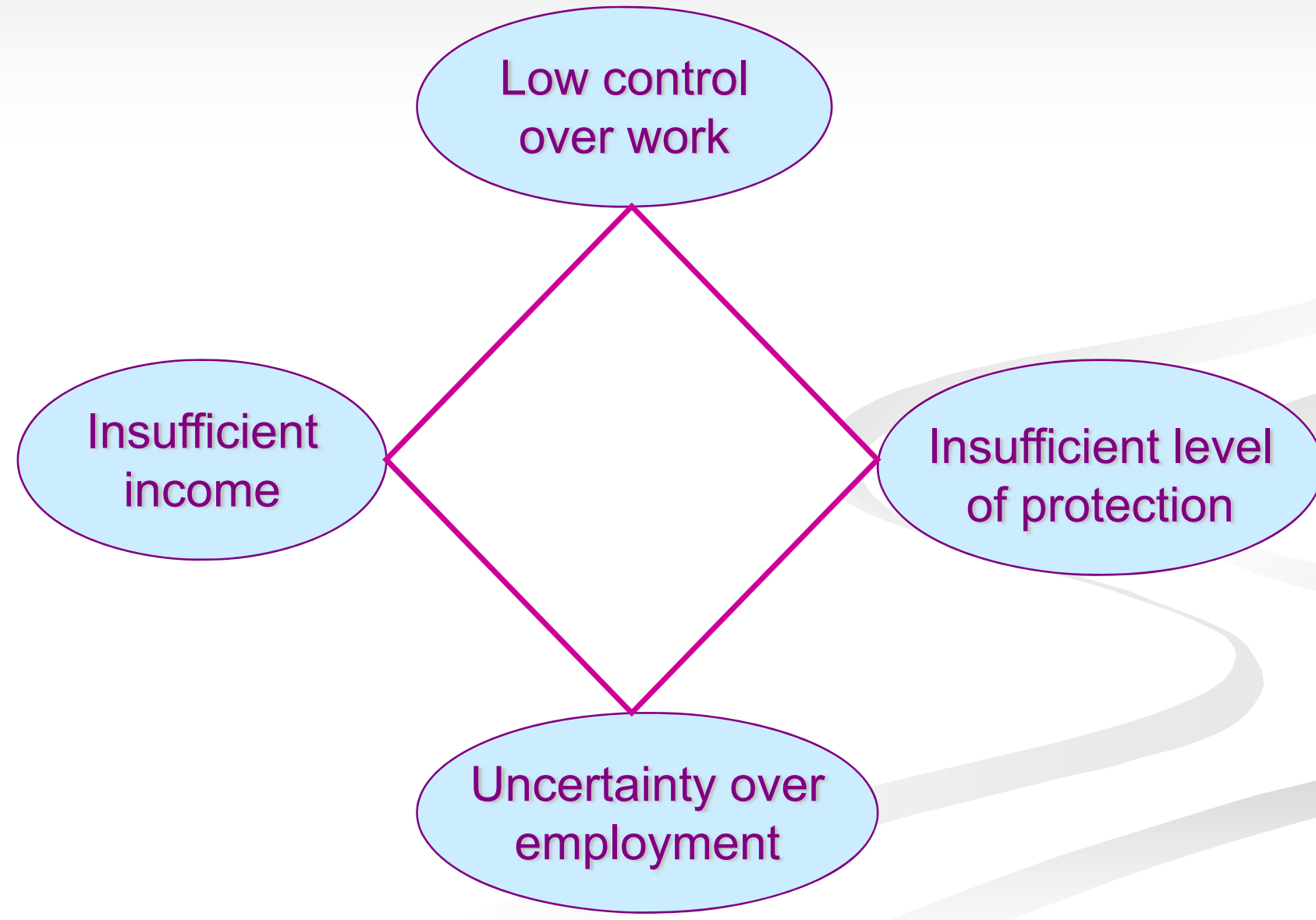




\section{THANKS}

Contacts

\section{belen.rando@ina.pt}

claudia.anjos@ina.pt

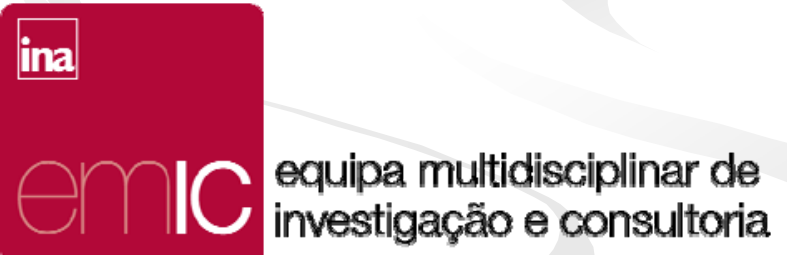

